\title{
In vivo confocal microscopy of ocular surface squamous neoplasia
}

R Parrozzani' ${ }^{1}$, D Lazzarini' ${ }^{1}$ A Dario ${ }^{2}$ and E Midena ${ }^{1,2}$

\begin{abstract}
Purpose To analyse in vivo structural and cellular features of ocular surface squamous neoplasia using clinical confocal microscopy. Methods Ten consecutive cases of untreated ocular surface squamous neoplasia were in vivo investigated using clinical confocal microscopy (ConfoScan4, Nidek Co. Ltd, Gamagori, Japan) with a $\times 40$ surface non-contact objective lens. Confocal microscopy images were compared with cytologic samples obtained by scraping technique.
\end{abstract}

Results Confocal microscopy examination revealed large areas of superficial cells debris and/or keratin debris accompanied by syncytial-like groupings, loss of the normal structure of the conjunctival epithelium and $\backslash$ or of the corneal basal epithelium layer, papillomatous organization, large fibrovascular structures, and fine vessels perpendicular to the tumour surface. Sub-epithelial (pre-Bowman) space involvement was documented in four cases $(50 \%)$. Irregular healthy tissue infiltration at the lateral edge of the lesion was documented in two cases $(20 \%)$ whereas abrupt demarcation between neoplastic cells and normal epithelium was documented in eight cases $(80 \%)$. In vivo cyto-morphologic study using clinical confocal microscopy showed cellular anisocytosis, pleocytosis, and anisonucleosis, enlarged nuclei with high nuclear to cytoplasmic ratio, high reflective cytoplasm and indistinct cytoplasmic borders in all cases $(\mathbf{1 0 0 \%})$.

Conclusion CCM appears to be a promising and non-invasive method for in vivo structural and cellular analysis of OSSN.

Eye (2011) 25, 455-460; doi:10.1038/eye.2011.11; published online 11 February 2011

Keywords: confocal microscopy; conjunctiva; carcinoma; neoplasia; cytology; in vivo.
Introduction

The spectrum of (corneo-conjunctival) ocular surface squamous neoplasia (OSSN) ranges from mild to severe epithelial dysplasia, through full thickness epithelial involvement, to invasive squamous cell carcinoma. ${ }^{1}$ The main treatment modality for these lesions should be wide local excision and accurate histological assessment of surgical margins. ${ }^{2}$ Moreover, topical conjunctival chemotherapy with antineoplastic drugs has recently been proposed in OSSN management, as adjuvant or, in selected cases, sole treatment. ${ }^{2-3}$ Diagnosis and follow-up of OSSN are essentially based on clinical findings followed by histopathologic confirmation. $^{2}$ Tissue diagnosis is commonly performed by excisional biopsy in smaller lesions, or map biopsy in multifocal-larger lesions. ${ }^{1,2}$ An alternative tool for tissue diagnosis is cytologic sampling using scraping or impression technique. ${ }^{1,4,5}$ Cytologic confirmation may be extremely valuable in diagnosing and monitoring OSSN, particularly when treated with topical chemotherapy. 1,2,4,5 Unfortunately, cytologic sampling may be uncomfortable for the patient and requires an experienced pathologist to interpret the specimens. ${ }^{1}$

Clinical confocal microscopy (CCM) obtains in vivo high-resolution optical images of human corneal layers and conjunctiva. ${ }^{6-8}$ The key features of CCM is its ability to produce in-focus images of thin slices $(5-20 \mu \mathrm{m})$ within a maximum depth of $1000 \mu \mathrm{m}$, a process known as optical sectioning. ${ }^{8}$ The aim of this study was to analyse in vivo structural and cellular features of OSSN using clinical confocal microscopy.

\section{Patients and methods}

This study complied with the tenets of the Declaration of Helsinki and was approved by the IRB of our Institutions. We certify that all
${ }^{1}$ Fondazione GB Bietti per I'Oftalmologia, IRCCS, Roma, Italy

${ }^{2}$ Department of Ophthalmology, University of Padova, Padova, Italy

Correspondence: E Midena, Department of Ophthalmology, University of Padova, Via Giustiniani 2 Padova 35128, Italy Tel: + 390498212110 ; Fax: + 390498212129 . E-mail: edoardo.midena@ unipd.it

Received: 11 October 2010 Accepted in revised form: 21 December 2010 Published online: 11 February 2011 
applicable institutional and governmental regulations concerning the ethical use of human volunteers were followed during this research. Each patient was recruited from those referred to the Ophthalmic Oncology Units of both participant Institutions and underwent a baseline full ophthalmologic examination. Clinical and demographic characteristics were collected, including: age, gender, tumour location (cornea, conjunctiva, and corneo-conjunctival), and tumour largest basal diameter. Adjunctive tumour clinical features, such as nodularity, mutifocality, prominent vascularisation (presence of macroscopically evident tumour vessels or conjunctival feeder vessels), and fornix involvement were also reported. ${ }^{9}$ Tumour clinical aspect was documented by anterior segment photography. Scraping cytology specimens were obtained at baseline from all patients. Cytologic analysis was reported as low-grade dysplasia (cells with enlarged nuclei, hyperchromasia, and irregular contour of the nuclear membrane with increased nuclear/cytoplasmic ratio), and high-grade dysplasia (pleomorphism of the nucleus with dyskeratotic cells). ${ }^{1,10}$ The presence of syncytial sheath, nucleoli, and infiltration of inflammatory cells was reported as invasive SCC. ${ }^{1,10}$ Scraping cytology results were confirmed by histopathologic examination in all cases. To be included in this study, each patient needed to be affected by untreated, clinically suspected, and cytologically confirmed OSSN, without clinical evidence of intraocular or orbital spread, aged 21 years or older and planned to undergo surgical excision as first treatment. Ten consecutive cases of OSSN were included in this case-series.

\section{Confocal microscopy analysis}

All tumours were investigated using in vivo clinical corneo-conjunctival confocal microscopy (ConfoScan4) with a $\times 40$ surface non-contact objective lens (Achroplan $40 \times / 0.75 \mathrm{~W}$, Zeiss, Oberkochen, Germany). This instrument has a field of view of $340 \times 255 \mu \mathrm{m}$, with a lateral resolution of $0.6 \mu \mathrm{m} / \mathrm{pix}$ and an optical slice thickness (z-axis resolution) of 5-20 $\mu \mathrm{m}$ within a maximum depth of $1000 \mu \mathrm{m}$. The objective lens has a working distance of $1.98 \mathrm{~mm}$, a numerical aperture of 0.75 and a front area of $16.61 \mathrm{~mm}^{2}$. Before examination, a drop of topical anaesthetic (Ossibuprocain, Novesina, Novartis Farma, Origgio, Italy) was applied, and a drop of methylcellulose solution (Viscotirs Gel, CIBA Vision Ophthalmics, Venice, Italy) was placed onto the tip of objective lens, as an immersion fluid. The objective lens was carefully aligned to the centre of the lesion and then, when technically possible, to the lesion margins (superior, nasal, inferior, and temporal margins) and to the paralesional area (superior, nasal, inferior, and temporal area). The objective lens was always monitored so that it never came into direct physical contact with the tumour surface. Confocal microscopy analysis was performed as continuous non-contact $\mathrm{z}$-axis scan of the lesion using manual and $\backslash$ or semiautomatic mode. During confocal microscopy scanning, the image focal plane was advanced at a speed of $\sim 25$ frames/s. Each scan was obtained in a mean of $15 \mathrm{~s}$ (range 7-20 s).

The main magnification obtained was $\times 500$ on a $15^{\prime \prime}$ display $(1024 \times 768$ pixels $)$.

\section{Intra- and inter-examiner reproducibility}

To check for intra-examiner reproducibility of each CCM investigation, each tumour feature (see Table 2) was analysed two times by the first operator; the second masked examination was performed 4 weeks apart. A second masked operator also analysed each feature (CCM images) to check for inter-examiner reproducibility.

\section{Results}

Ten consecutive patients were included in this pilot study. Mean tumour largest basal diameter was $8.3 \pm 3.1 \mathrm{~mm}$ (range 3-13.0). Two tumours (20\%) were conjunctival in location and eight were corneoconjunctival (80\%). Baseline scraping cytology results, confirmed by histopathologic examination, are reported in Table 1. Corneo-conjunctival confocal microscopy analysis showed structural (tissue architecture level), marginal (tumour margins), and cyto-morphologic (cell morphology level) features of the lesions.

\section{Structural findings}

Structural aspects were: large areas of superficial cells debris and/or keratin debris accompanied by syncytiallike groupings (Figure 1a), loss of the normal structure of the conjunctival epithelium, and \or of the corneal basal epithelium layer (Figure 1b), papillomatous organization (Figure 1c), large fibrovascular structures (Figure 1d), and fine vessels perpendicular to the tumour surface (Figure 1e). Confocal microscopy findings related to cytologic results are reported in Table 2.

\section{Marginal findings}

\section{Depth of invasion}

Corneal sub-epithelial space and anterior stroma examination through the tumour centre was possible in 5 of 8 tumours $(62.5 \%)$, because of tumour thickness ( $>1000 \mu \mathrm{m}$ ), and in all tumours through the tumour margins (100\%). Sub-epithelial (pre-Bowman) space involvement was documented in four cases $(50 \%)$ 
(Figure 3a). Anterior stroma was normal in all cases, including the lesions with sub-epithelial involvement, without any evidence of tumour infiltration (Figure 3a).

\section{Lateral margins}

Irregular healthy tissue infiltration at the lateral edge of the lesion was documented in two cases $(20 \%)$, whereas

Table 1 Demographic and clinical data of examined eyes

\begin{tabular}{|c|c|c|c|c|c|c|c|c|c|c|c|}
\hline No. & Age & Gender & Eye & $L B D(m m)$ & $\begin{array}{l}\text { Anatomic } \\
\text { location }\end{array}$ & $\begin{array}{c}\text { Meridian } \\
\text { location }\end{array}$ & Nodularity & Vascularity & Multifocality & $F I$ & Histopathology \\
\hline 1 & 73 & M & $\mathrm{R}$ & 6 & $\mathrm{CC}$ & $\mathrm{N}$ & & & & & LGD \\
\hline 2 & 65 & $\mathrm{M}$ & $\mathrm{L}$ & 8 & $\mathrm{CC}$ & $\mathrm{I}, \mathrm{N}$ & + & + & & & HGD \\
\hline 3 & 61 & M & $\mathrm{R}$ & 5 & $\mathrm{CC}$ & $\mathrm{T}$ & & + & & & SCC \\
\hline 4 & 70 & $\mathrm{~F}$ & $\mathrm{R}$ & 3 & $\mathrm{CC}$ & $\mathrm{T}$ & & & & & HGD \\
\hline 5 & 70 & $\mathrm{~F}$ & $\mathrm{R}$ & 4 & Conjunctiva & $\mathrm{T}, \mathrm{S}$ & & & & & LGD \\
\hline 6 & 79 & M & $\mathrm{R}$ & 3 & Conjunctiva & $\mathrm{N}, \mathrm{I}$ & & & & & LGD \\
\hline 7 & 68 & $\mathrm{M}$ & $\mathrm{R}$ & 13 & $\mathrm{CC}$ & $\mathrm{N}, \mathrm{S}, \mathrm{I}$ & + & + & + & + & SCC \\
\hline 8 & 72 & $\mathrm{M}$ & $\mathrm{L}$ & 9 & $\mathrm{CC}$ & $\mathrm{T}, \mathrm{S}$ & + & & & & SCC \\
\hline 9 & 80 & F & $\mathrm{L}$ & 12 & $\mathrm{CC}$ & $\mathrm{N}, \mathrm{I}$ & & + & & + & SCC \\
\hline 10 & 77 & M & $\mathrm{L}$ & 11 & $\mathrm{CC}$ & $\mathrm{T}, \mathrm{I}$ & + & + & & & HGD \\
\hline
\end{tabular}

Abbreviations: CC, cornea and conjunctiva; F, female; FI, fornix involvement; HGD, high grade dysplasia; I, inferior; L, left; LBD, largest basal diameter; LGD, low grade dysplasia; M, male; N, nasal; R, right; S, superior; SCC, invasive squamous cell carcinoma; $T$, temporal.
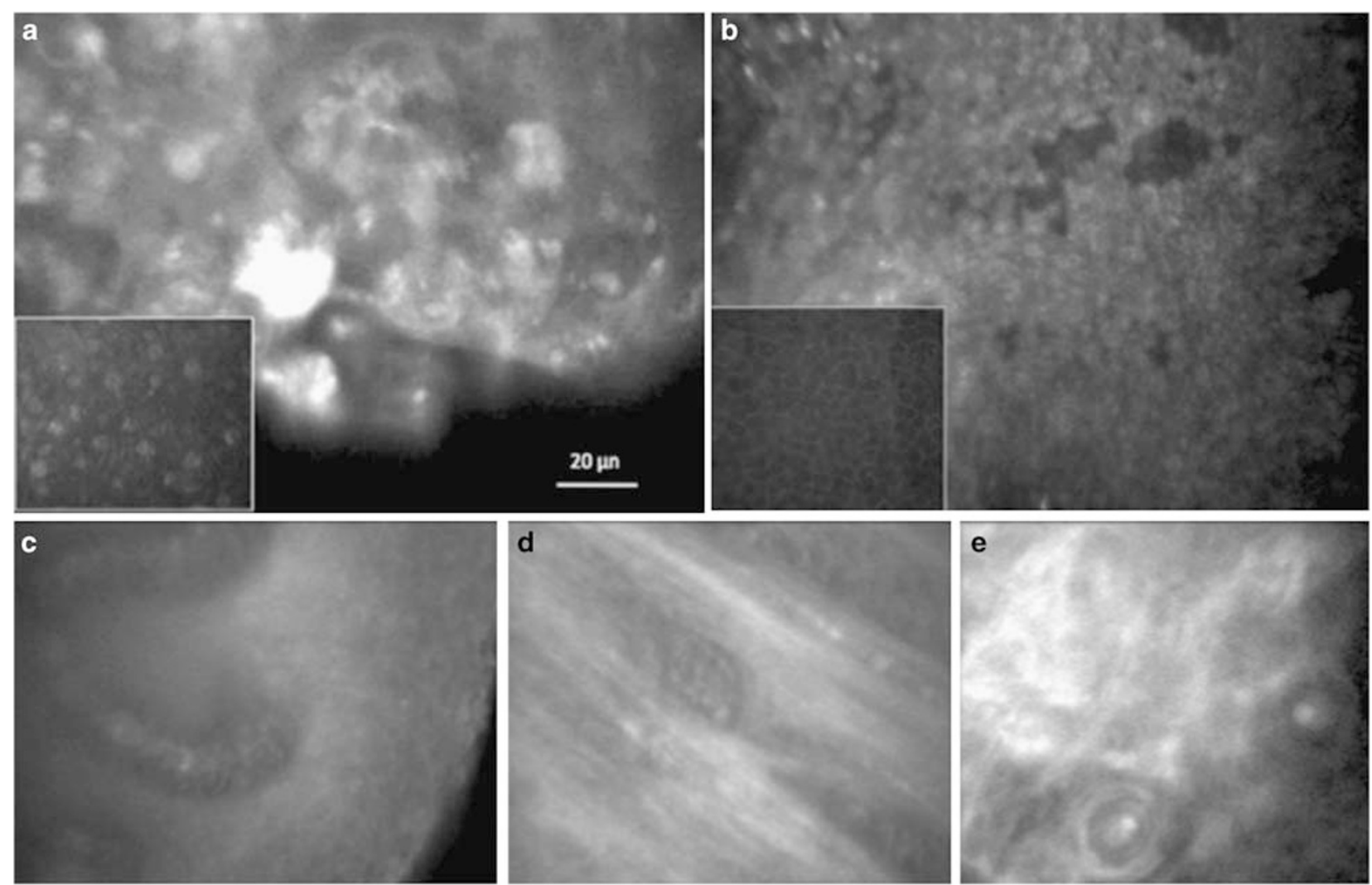

Figure 1 Loss of the normal corneal epithelium structure in high-grade dysplasia (a). (Top right) confocal microscopy analysis shows large areas of superficial cells debris accompanied by syncytial-like groupings with well-visible atypical cells. (Bottom left) Normal corneal epithelium. Loss of the normal conjunctival epithelium structure in low-grade dysplasia (b). (Top right) confocal microscopy analysis shows small, atypical, high-reflective round cells. (Bottom left) Normal conjunctival epithelium. Papillomatous organization (note the radial vascular core) (c), large intralesional fibrovascular structures (d) and fine vessels perpendicular to the tumour surface (note vessels lumen and wall) in different lesions (e). 
Table 2 In vivo confocal microscopy of ossn: structural, marginal and cyto-morphological findings related to cytological diagnosis

\begin{tabular}{|c|c|c|c|}
\hline \multirow{2}{*}{ In vivo confocal microscopy findings } & \multicolumn{3}{|c|}{ Histopathology } \\
\hline & Low-grade dysplasia & High-grade dysplasia & SCC \\
\hline Cells debris and/or keratin debris & $0 / 3$ & $3 / 3$ & $4 / 4$ \\
\hline Syncytial-like groupings & $0 / 3$ & $3 / 3$ & $4 / 4$ \\
\hline Structural abnormalities of conjunctival and/or corneal basal epithelium layer & $3 / 3$ & $3 / 3$ & $4 / 4$ \\
\hline Fine vessels perpendicular to the tumour surface & $0 / 3$ & $0 / 3$ & $2 / 4$ \\
\hline Large fibrovascular structures & $0 / 3$ & $1 / 3$ & $3 / 4$ \\
\hline Papillomatous organization & $1 / 3$ & $1 / 3$ & $1 / 4$ \\
\hline Sub-epithelial space involvement & $0 / 3$ & $0 / 3$ & $4 / 4$ \\
\hline Anterior stroma involvement & $0 / 3$ & $0 / 3$ & $0 / 4$ \\
\hline Demarcation at the lateral edge of the lesion & $3 / 3$ & $2 / 3$ & $3 / 4$ \\
\hline Irregular margins infiltration & $0 / 3$ & $1 / 3$ & $1 / 4$ \\
\hline Cellular anisocytosis & $3 / 3$ & $3 / 3$ & $4 / 4$ \\
\hline Cellular pleocytosis & $3 / 3$ & $3 / 3$ & $4 / 4$ \\
\hline Cellular anisonucleosis & $3 / 3$ & $3 / 3$ & $4 / 4$ \\
\hline Enlarged and polarised nuclei & $3 / 3$ & $3 / 3$ & $4 / 4$ \\
\hline High reflective cytoplasm & $3 / 3$ & $3 / 3$ & $4 / 4$ \\
\hline Indistinct cytoplasmic border & $3 / 3$ & $3 / 3$ & $4 / 4$ \\
\hline
\end{tabular}

abrupt demarcation between neoplastic cells and normal epithelium was present in eight cases (80\%) (Figure $3 b$ )

\section{Cyto-morphological findings}

In vivo cyto-morphologic study of the tumour using clinical confocal microscopy was feasible in all 10 tumours (100\%). Cellular anisocytosis, pleocytosis, and anisonucleosis, enlarged, and polarised nuclei with high nuclear to cytoplasmic ratio, high-reflective cytoplasm, and indistinct cytoplasmic borders were documented in all cases (100\%) (Figure 1a, Figure 3). In vivo CCM analysis showed well visible dysplastic cells in each analysed tumour, showing morphologic agreement with ex vivo scraping cytology and histology in all cases (100\%) (Figure 2a, Figure 3).

\section{Intra- and Inter-examiner reproducibility}

Excellent agreement was found for both intra-examiner reproducibility $(98.2 \%)$, and inter-examiner reproducibility $(96.3 \%)$ for each tumour feature reported in Table 2.

\section{Discussion}

In vivo evaluation of the ocular structures at high magnification (to distinguish microscopic cell details) has always been a challenge for ophthalmic clinicians and researchers, but microscopic studies have, until recently, been limited to ex vivo investigations. ${ }^{8}$ Clinical biomicroscopy and pathologic examination of sampled specimens continue to have the major role in diagnosing and monitoring OSSN. Unfortunately, biomicroscopy is limited by low magnification and needs histo- or cyto-pathologic confirmation. ${ }^{1,2}$ Confocal microscopy was introduced into the clinical practice as a noninvasive tool to observe in vivo, at high magnification, the structures of human cornea and conjunctiva. ${ }^{6,7}$ CCM analysis extends the principles of biomicroscopy to the microscopic range, scanning the examined tissue layer by layer (5-20 $\mu \mathrm{m}$ 'slices') by changing the plane of focus of the detector source. ${ }^{6-8}$

Only few data were reported about CCM analysis of ocular surface tumours. ${ }^{11-13}$ Duchateau et al ${ }^{11}$ reported three cases of OSSN analysed with CM, concluding that this method could be a diagnostic aid especially for clinically atypical lesions. The main pathological features were: cytonuclear atypias, epithelial folds into an inflammatory and vascularised conjunctival stroma, fine vessels perpendicular to the surface, clear limit with normal epithelium, papillomatous organization, and abnormal keratinisation. ${ }^{11}$ Our results confirm, in a larger series, these findings. Messmer et $a l^{12}$ analysed 28 pigmented conjunctival tumours using CCM, including two extrascleral growths of uveal melanoma. CCM analysis showed high sensitivity and specificity for diagnosing melanocytic lesions of the conjunctiva compared with standard histopathology. ${ }^{12}$ More recently, Pichierri et $a l^{13}$ reported one case of conjunctival lymphoma analysed using CCM, concluding that CCM imaging recalled the histological profile of low-grade mucosa-associated lymphoid tissue lymphoma. Gentile $e t \mathrm{al}^{14}$ reported seven eyes affected by corneal intraepithelial neoplasia, documenting pleomorphic, medium sized, hypereflective nucleated cells with indistinct cytoplasmic border, with a sharp transition between neoplastic, and non-neoplastic epithelium. In our series, structural and cellular analysis using CCM 


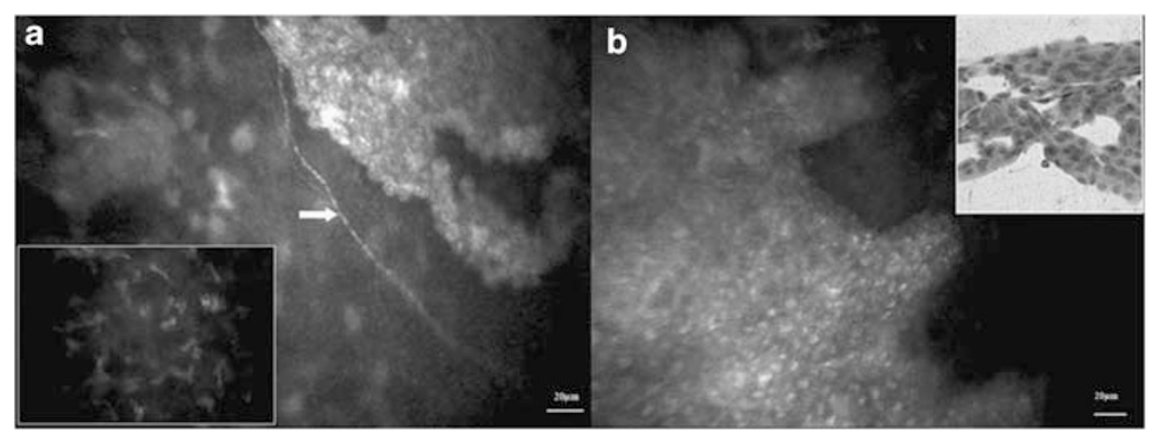

Figure 2 Pre-Bowman involvement in a case of squamous cell carcinoma (a). (Top-right) Confocal microscopy analysis shows small atypical high-reflective round cells near a subbasal nerve fibre (arrow). (Bottom-left) Normal anterior stroma was found just behind the pre-bowman involvement $(5 \mu \mathrm{m})$. Conjunctival epithelium infiltration by tumour cells (b). (Top right) Abrupt demarcation at the lateral edge of the lesion between neoplastic cells and uninvolved benign conjunctival epithelium in a case of low-grade dysplasia. (Bottom left) Cytologic aspect of the same lesion shows monomorphic, squamous cells with some irregularities of nuclei (Papanicolaou, $\times 240$ ).

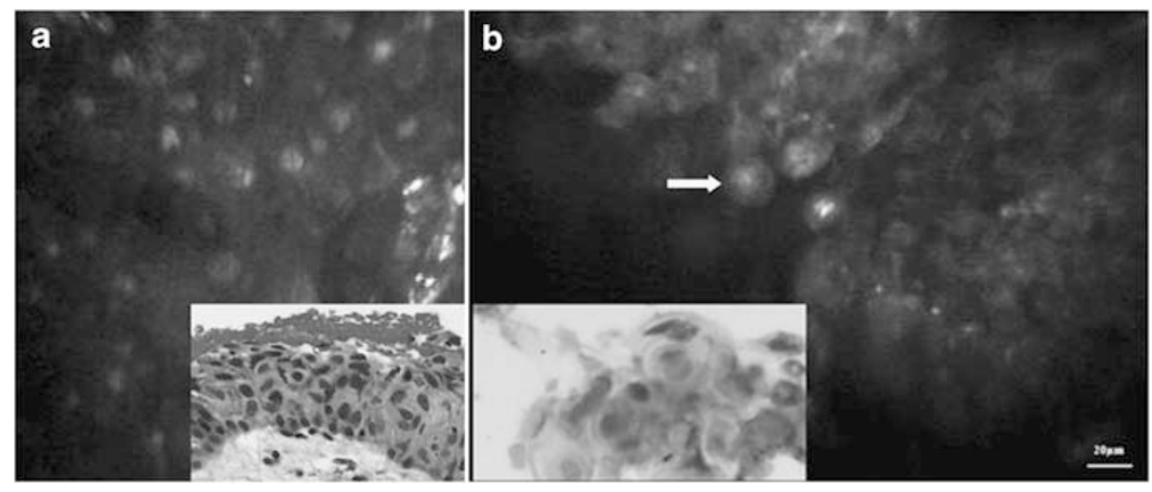

Figure 3 Confocal microscopy aspect in a case of high-grade dysplasia (a). (Top left) Note the diffuse nuclear enlargement and the irregular nuclear shape. (Bottom right) Histological aspect of the same lesion (Papanicolaou, $\times 150$ ). Scraping cytology and confocal microscopy aspect in a case of squamous cell carcinoma (b). (Top right) Confocal microscopy shows atypical cells with high nuclear to cytoplasmic ratio, high-reflective cytoplasm, indistinct cytoplasmic and nuclear border (arrow). (Bottom left) Scraping cytology aspect of the same case (Papanicolaou, $\times 240$ ).

showed well visible dysplastic cells in each tumour, confirming full morphologic agreement with ex vivo scraping cytology in all cases. Moreover, CCM showed corneal sub-epithelial space involvement in four cases, confirming in vivo the diagnosis of invasive SCC.

Barros et $a l^{15}$ recently reported an index score to differentiate SCC from pre-invasive ocular surface lesions. Four of seven parameter included in this regression model (nuclear enlargement $>\times 3$, syncytiallike groupings, increased nuclear-to-cytoplasmic ratio, and indistinct cytoplasm border) are clearly visible using CCM. One parameter (prominent nucleoli) is currently undetectable by CCM. The last two parameters (cellular hyperchromasia and eosinophylic cytoplasm) need specific stains unavailable in vivo. The introduction of in vivo stains or biomarkers to better underline these cell detail will be useful to improve image quality and to obtain more detailed information. ${ }^{15}$ Moreover,
Mocan $e t a l^{16}$ recently reported the in vivo analysis of corneal epithelium using fluorescein-enhanced CCM, concluding that fluorescein is able to enhance the visualisation of superficial corneal epithelium and may be used to evaluate this layer to a greater extent both quantitatively and qualitatively.

Nevertheless, other parameters (not included in the logistic regression by Barros et al, but commonly used in standard histopathology) are easily detectable using CCM, mainly both structural aspects (syncytial-like groupings, loss of the normal structure of the conjunctival epithelium and $\backslash$ or of the corneal basal epithelium layer) and tumour margins (pre-Bowman involvement). ${ }^{5}$ The introduction of a new CCM specific index score to differentiate SCC from pre-invasive ocular surface lesions is underway.

The high value of CCM technique results on: ability to detect both the presence and extent of OSSN, when 
the clinical diagnosis is difficult; to detect subclinical disease and to follow-up previously diagnosed disease with a minimally invasive procedure. Moreover, excellent agreement was found as intra- and inter-operator reproducibility is concerned.

The quality of the CCM images depends on the illumination of the focused object and light reflected by it. ${ }^{11,17}$ Because of its organization, normal cornea, and conjunctiva are purely transparent structures. ${ }^{1,11,17}$ On the contrary, the structure of keratinised, hypoxic, and partially necrotic neoplastic proliferation has not the same favourable optic properties. ${ }^{11}$ Therefore, clinical confocal microscopy interpretation may be more difficult in corneal/conjunctival tumours, when tumour surface clinically appears leukoplakic. In these cases, CCM analysis and imaging quality may be improved performing CCM after a gentle superficial debridement, aimed at removing superficial high reflective keratinised cells. In conclusion, CCM of the ocular surface seems a promising tool for in vivo non-invasive microscopic imaging of OSSN. The introduction of this technique in a routine clinical setting may improve in vivo characterisation of OSSN, moving clinical diagnosis from slit lamp magnifications into a microscopic magnification.

\section{Summary}

What was known before

- Diagnosis and follow-up of OSSN are essentially based on clinical findings followed by histopathologic confirmation.

\section{What this study adds}

- The introduction of this technique in a routine clinical setting may improve in vivo characterisation of ocular surface squamous tumour, moving clinical diagnosis from slit lamp magnifications into a microscopic magnification.

\section{Conflict of interest}

The authors declare no conflict of interest.

\section{Acknowledgements}

This study was supported by Fondazione GB Bietti per l'Oftalmologia, IRCCS, Via Livenza 3, 00198, Roma, Italy.

\section{Disclaimer}

The correspondent author had full access to all the data in the study and takes responsibility for the integrity of the data and the accuracy of the data analysis.

\section{References}

1 Lee GA, Hurst LW. Ocular surface squamous neoplasia. Surv Ophthalmol 1995; 39: 429-450.

2 Midena E, Degli Angeli C, Valenti M, de Belvis V, Boccato P. Treatment of conjunctival squamous cell carcinoma with topical 5-fluorouracil. Br J Ophthalmol 2000; 84: 268-272.

3 Midena E, Boccato P, Angeli CD. Conjunctival squamous cell carcinoma treated with topical 5-fluoruracil. Arch Ophthalmol 1997; 115: 1600-1601.

4 Tole DM, McKelvie PA, Daniell M. Reliability of impression cytology for the diagnosis of ocular surface squamous neoplasia employing the Biopore membrane. $\mathrm{Br} \mathrm{J}$ Ophthalmol 2001; 85: 154-158.

5 Nolan GR, Hirst LW, Bancroft BJ. The cytomorphology of ocular surface squamous neoplasia by using impression cytology. Cancer 2001; 93: 60-67.

6 Chiou AGY, Kaufman SC, Kaufman HE, Beuerman RW. Clinical corneal confocal microscopy. Surv Ohphthalmol 2006; 51: 482-500.

7 Zhivov A, Stachs O, Kraak R, Stave J, Guthoff RF. In vivo confocal microscopy of the ocular surface. Ocul Surf 2006; 4: 81-93.

8 Böhnke M, Masters BR. Confocal microscopy of the cornea. Prog Retin Eye Res 1999; 18: 553-628.

9 Parrozzani R, Lazzarini D, Alemany-Rubio E, Urban F, Midena E. Topical 1\% 5-fluoruracil in ocular surface squamous neoplasia: a long-term safety study. $\mathrm{Br} J$ Ophthalmol 2010; E-pub ahead of print 7 August 2010; doi:10.1136/bjo.2010.183244.

10 Tananuvat N, Lertprasertsuk N, Mahanupap P, Noppanakeepong P. Role of impression cytology in diagnosis of ocular surface neoplasia. Cornea 2008; 27: 269-274.

11 Duchateau N, Hugol D, D’Hermies F, Meyer A, Labbé A, Dupas B et al Contribution of in vivo confocal microscopy to limbal tumor evaluation. J Fr Ophtalmol 2005; 28: 810-816.

12 Messmer EM, Mackert MJ, Zapp DM, Kampik A. In vivo confocal microscopy of pigmented conjunctival tumors. Graefes Arch Clin Exp Ophthalmol 2006; 244: 1437-1445.

13 Pichierri P, Martone G, Loffredo A, Traversi C, Polito E. In vivo confocal microscopy in a patient with conjunctival lymphoma. Clin Experiment Ophthalmol 2008; 36: 67-69.

14 Gentile CM, Croxatto JO. In vivo diagnosis of epithelial neoplastic changes of the cornea and conjunctival limbus using confocal microscopy. ARVO Meeting Abstracts 11 April 2008; 49: 5680.

15 Barros JN, Lowen MS, Ballalai PL, Mascaro VLDM, Gomes JAP, Martins MC. Predictive index to differentiate invasive Squamous cell carcinoma from preinvasive ocular surface lesions by impression cytology. $\mathrm{Br} J$ Ophthalmol 2009; 93: 209-214.

16 Mocan MC, Irkec M. Fluorescein enhanced confocal microscopy in vivo for the evaluation of corneal epithelium. Clin Experiment Ophthalmol 2007; 35: 38-43.

17 Midena E, Gambato C, Miotto S, Cortese M, Salvi R, Ghirlando A. Long-term effects on corneal keratocytes of mitomycin $\mathrm{C}$ during photorefractive keratectomy: a randomized contralateral eye confocal microscopy study. J Refract Surg 2007; 23: S1011-S1014. 